\title{
Effect of Vascular Comorbidities on Survival of Type 2 Diabetes Patients on Renal Replacement Therapy
}

\author{
Marjo Kervinen $^{a}$ Seppo Lehto ${ }^{a} \quad$ Carola Grönhagen-Riska ${ }^{b, c}$ Patrik Finne ${ }^{b, c}$ \\ ${ }^{a}$ Department of Internal Medicine, Kuopio University Hospital, Kuopio, ${ }^{b}$ Finnish Registry for Kidney Diseases, and \\ 'Division of Nephrology, Department of Medicine, Helsinki University Central Hospital, Helsinki, Finland
}

\section{Key Words}

Diabetes mellitus - Renal replacement therapy · Survival

\begin{abstract}
Background: Atherosclerosis is an important predictor of mortality in patients with end-stage renal disease. The aim of this study was to determine how various vascular comorbidities such as coronary heart disease (CHD), peripheral vascular disease (PVD) or cerebrovascular disease (CeVD) affect survival of type 2 diabetic patients on renal replacement therapy (RRT). Methods: Patients who entered RRT because of type 2 diabetes in 2000-2008 ( $n=877$ ) were identified within the Finnish Registry for Kidney Diseases. The patients were followed up until death or end of follow-up. Survival probabilities were calculated using Kaplan-Meier curves. Multivariate modeling was performed using Cox regression. Results: $41 \%$ of the patients had CHD, 27\% PVD and $16 \%$ CeVD at the start of RRT. Patients with PVD had a 1.9-fold (95\% Cl 1.6-2.3) risk of death compared to those without PVD when adjusting for age and gender, while patients with CHD had a 1.5-fold (95\% Cl 1.2-1.8) and those with CeVD a 1.4-fold (95\% Cl 1.1-1.8) risk compared to those without these diseases. The hazard ratio (HR) for death was highest in patients with the combination of PVD and either CHD (HR 2.8, 95\%
\end{abstract}

Cl 2.1-3.8) or CeVD (HR 2.9, 95\% Cl 1.6-5.2) as compared to patients without any vascular comorbidities. Conclusion: PVD is the vascular comorbidity that increases risk of death the most among patients with type 2 diabetes starting RRT. Prevention of PVD in this patient group would merit further studies.

Copyright $\odot 2012$ S. Karger AG, Basel

\section{Introduction}

Vascular calcification is an important predictor of allcause mortality in patients with end-stage renal disease (ESRD) [1]. Furthermore, patients with diabetes as the cause of ESRD have higher overall and cardiovascular mortality than non-diabetic patients [2]. There are two distinct forms of vascular calcification: intimal atherosclerosis narrows vessels, whereas medial calcification stiffens them and increases pulse wave velocity and cardiac afterload. Both intimal and medial calcifications are associated with increased risk of cardiovascular death in dialysis patients [3]. Aortic stiffness increases both allcause and cardiovascular mortality among ESRD patients with type 2 diabetes [4].

\section{KARGER \\ Fax +41613061234 E-Mail karger@karger.ch} www.karger.com

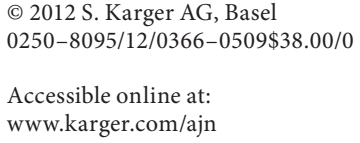

Marjo Kervinen, MD

Department of Internal Medicine, Dialysis Unit, Kuopio University Hospital

PL 1777

FI-70211 Kuopio (Finland)

E-Mail marjo.kervinen@kuh.fi 
Although patients with type 2 diabetes commonly develop albuminuria and decreased creatinine clearance, only a very small proportion develops ESRD mainly because death is a frequent competing risk event $[5,6]$. Villar et al. [7] found that ESRD is associated with a relatively smaller increase in mortality in patients with than without type 2 diabetes. This may be due to ESRD increasing risk of cardiovascular disease relatively less in type 2 diabetes than in non-diabetes patients. Nonetheless, type 2 diabetes patients with ESRD had twofold overall mortality compared to ESRD patients without diabetes. Lok et al. [8] reported that diabetes patients had more comorbidities than other patients entering dialysis, and speculated that this may contribute to their relatively high mortality. This finding has recently been confirmed by Helve et al. [9] on ESRD patients with type 1 diabetes. Our study is the first one performed solely on type 2 diabetes patients on renal replacement therapy (RRT) estimating the effect of vascular comorbidities on survival.

The primary aim of this study was to determine how the vascular comorbidities coronary heart disease (CHD), peripheral vascular disease (PVD) and cerebrovascular disease (CeVD) affect survival of type 2 diabetes patients on RRT. Our study population is uniquely suitable for this purpose as it is a nationwide incidence cohort with virtually complete coverage of type 2 diabetes patients entering RRT in Finland. In addition, we have the possibility to adjust for most potential confounding factors, such as obesity, hypertension, hyperglycemia, serum lipid profile and other laboratory parameters. The secondary aim of our study was to assess frequency of vascular complications among type 2 diabetes patients at start of RRT.

\section{Subjects and Methods}

Patients who entered RRT in Finland because of type 2 diabetes mellitus in 2000-2008 were identified from the Finnish Registry for Kidney Diseases. This registry is maintained by the Finnish Kidney and Liver Organization and is supported by Finnish governmental grants. The registry has been estimated to cover 97-99\% of all RRT patients in Finland [10]. The study was approved by the Ethics Committee of the University of Kuopio and the board of the Finnish Registry for Kidney Diseases.

The registry contains information at start of RRT on vascular comorbidities, heart failure, body-mass index (BMI), serum lipid profile, glycated hemoglobin (HbAlc), phosphorus, albumin, systolic and diastolic blood pressure, previous and present smoking. In addition, the registry stores follow-up data, e.g. on kidney transplantations and types of vascular access. The registry col- lects data on CHD using three variables: (1) angina pectoris, (2) myocardial infarction in the past and (3) history of coronary bypass surgery or angioplasty. In this study, the patients were considered to have CHD if at least one of these variables was positive. Three variables on PVD were available in the registry: (1) symptomatic PVD, (2) surgery because of PVD, (3) amputation because of PVD. Patients in this study were considered to have PVD if at least one of these variables was positive. CeVD included cerebral infarctions and hemorrhages. The patients with incomplete data on vascular comorbidities were not included in the study. Causes of death were available as ICD-10 codes and were analyzed in four groups: cardiovascular including all cardiac and vascular causes, infection or other cause except kidney disease itself and kidney disease.

Basic patient characteristics were compared between groups using the Mann-Whitney U test for continuous variables and the $\chi^{2}$ test for categorical variables. Patients were followed up from start of RRT to death or to end of follow-up on 31 December 2008. Consequently, patients who entered RRT in later years had shorter follow-up time, but this did not bias estimates of survival probabilities or relative risks of death because survival times were censored at end of follow-up using the Kaplan-Meier method and Cox regression. Death was the event in these analyses. Differences in survival probabilities between groups were assessed using the log rank test. The multivariate modeling using Cox proportional hazards regression allowed adjusting for potential confounding factors. Statistical analyses were performed using SPSS version 17.0.

\section{Results}

During 2000-2008, 4,425 patients entered RRT in Finland. The cause of ESRD was type 2 diabetes in $20 \%$ ( $\mathrm{n}=$ 877 , included in this study), type 1 diabetes in $15 \%$ and other in $65 \%$. Among the type 2 diabetes patients, the median age at start of RRT was 66.5 years (range 31.088.1). Of the patients, $35 \%(303 / 877)$ were women. The median follow-up time was 1.93 years (interquartile range 0.93-3.57 years). By the end of study period, 504 patients had died (57\%). The cause of death was cardiovascular in $50 \%$, infection in $22 \%$, kidney disease itself (or possibly unknown cause of death) in $6 \%$ and other cause (mostly diabetes, cancer and gastrointestinal diseases) in $22 \%$ of the deceased. When studying the causes of death in the different vascular comorbidities, the cardiovascular cause was most common in patients with $\mathrm{CeVD}$ (61.8\% of the deceased CeVD patients), whereas infection was most common in patients with PVD (21.3\% of the deceased PVD patients). 356 patients had CHD at start of RRT, and of these 93 (26\%) died of a CHD event. Among those without CHD at start, 13\% (61/464) died of CHD. Similarly, 5\% (7/136) of patients with a history of stroke died of stroke, while 3\% (20/694) of patients without a history of stroke died of stroke. PVD was recorded as cause of 
Table 1. Characteristics of type 2 diabetes patients starting RRT in 2000-2008 in Finland

\begin{tabular}{|c|c|c|c|}
\hline Variable & $\begin{array}{l}\text { No vascular } \\
\text { comorbidities }\end{array}$ & $\begin{array}{l}\text { One vascular } \\
\text { comorbidity }\end{array}$ & $\begin{array}{l}\text { Two or more vascular } \\
\text { comorbidities }\end{array}$ \\
\hline Patients & 311 & 275 & 189 \\
\hline Age at start of RRT, years & $63.1 \pm 9.7$ & $67.8 \pm 9.0$ & $67.6 \pm 7.7$ \\
\hline Women/men & $109 / 202$ & $94 / 181$ & $62 / 127$ \\
\hline Patients with first kidney transplant, $\%$ & 12.5 & 5.1 & 0.5 \\
\hline Peritoneal dialysis/hemodialysis, $\mathrm{n}$ & $63 / 248$ & $45 / 230$ & $27 / 162$ \\
\hline Patients with present smoking, $\%$ & 15.1 & 9.1 & 14.8 \\
\hline Patients with previous smoking, $\%$ & 26.4 & 31.6 & 38.6 \\
\hline Patients with previous coronary surgery or angioplasty, \% & 0.0 & 24.0 & 37.0 \\
\hline $\begin{array}{l}\text { Patients with previous arteriosclerotic surgery or angioplasty of } \\
\text { the extremities, } \%\end{array}$ & 0.0 & 12.7 & 37.0 \\
\hline Patients with limb amputation, $\%$ & 0.0 & 16.4 & 25.4 \\
\hline Patients with decompensation of heart, $\%$ & 6.8 & 21.8 & 38.6 \\
\hline BMI & $31.0 \pm 6.8$ & $29.9 \pm 5.8$ & $29.6 \pm 5.3$ \\
\hline Systolic blood pressure, mm Hg & $159 \pm 25$ & $154 \pm 24$ & $146 \pm 26$ \\
\hline Diastolic blood pressure, $\mathrm{mm} \mathrm{Hg}$ & $81 \pm 12$ & $78 \pm 11$ & $75 \pm 13$ \\
\hline HbAlc, \% & $7.2 \pm 1.4$ & $7.3 \pm 1.4$ & $7.6 \pm 1.4$ \\
\hline Total cholesterol, mmol/l & $4.0 \pm 1.3$ & $3.8 \pm 1.3$ & $3.9 \pm 1.3$ \\
\hline HDL cholesterol, mmol/l & $1.1 \pm 0.4$ & $1.0 \pm 0.4$ & $1.0 \pm 0.4$ \\
\hline LDL cholesterol, mmol/l & $2.1 \pm 1.1$ & $1.9 \pm 1.0$ & $2.1 \pm 1.1$ \\
\hline Use of lipid-lowering drugs, \% & 64 & 67 & 76 \\
\hline Triglycerides, $\mathrm{mmol} / \mathrm{l}$ & $1.7 \pm 0.9$ & $1.8 \pm 1.2$ & $1.7 \pm 1.0$ \\
\hline C-reactive protein, $\mathrm{g} / \mathrm{l}$ & $19 \pm 36$ & $20 \pm 30$ & $29 \pm 46$ \\
\hline Hemoglobin, $g / 1$ & $107 \pm 15$ & $107 \pm 14$ & $109 \pm 15$ \\
\hline Phosphorus, mmol/l & $1.91 \pm 0.58$ & $1.85 \pm 0.52$ & $1.88 \pm 0.68$ \\
\hline I-calcium, mmol/l & $1.14 \pm 0.12$ & $1.13 \pm 0.12$ & $1.15 \pm 0.12$ \\
\hline Albumin, g/l & $31 \pm 7$ & $32 \pm 5$ & $32 \pm 6$ \\
\hline
\end{tabular}

Data are means $\pm \mathrm{SD}$, unless stated otherwise.

One vascular comorbidity: 1 of the vascular comorbidities CHD, PVD or CeVD. Two or more vascular diseases: 2 or 3 of the abovementioned vascular diseases. 775 out of $877(88 \%)$ had complete data on all vascular comorbidities and were included in the analysis.

death in 4\% (10/245) of patients with PVD and 2\% (13/558) of patients without PVD at start of RRT.

Of the patients, 33\% (292/877) had angina pectoris, $23 \%(205 / 877)$ had a history of myocardial infarction and $17 \%(145 / 877)$ had a history of coronary bypass operation or angioplasty, 41\% (356/877) had CHD (at least one of the aforementioned conditions), 18\% (162/877) had heart failure, 90\% (786/877) hypertension, 25\% (219/877) had symptomatic PVD, $12 \%(109 / 877)$ had a history of arteriosclerotic surgery, 11\% (94/877) had a history of limb amputation, and 28\% (245/877) had PVD altogether (at least one of the three aforementioned conditions) and $16 \%(136 / 877)$ had a history of CeVD at start of RRT. Of the patients, $6 \%$ lacked data on CHD, $8 \%$ on PVD and $5 \%$ on CeVD. Data on all three main vascular comorbidities were available for 775 (88\%) patients, and these were included in the analyses of vascular comorbidities. $79 \%$ of patients had data on smoking; of these, 15\% (107/697) were current and 39\% (268/694) previous smokers at the start of RRT. Patient characteristics grouped by the number of vascular comorbidities are shown in table 1 .

The median survival of all patients was 3.0 years (range 0.2-8.7, 95\% CI 2.7-3.3).

The median survival was 2.4 years of patients with $\mathrm{CHD}$ and 3.8 years of patients without CHD $(\mathrm{p}<0.001)$. The age- and gender-adjusted hazard ratio (HR) for death was 1.48 for patients with CHD compared to those without (table 2). Patients with CHD were older, and they received a kidney transplant less frequently (3 vs. 10\%) than patients without CHD. They also had more often PVD and CeVD than patients without CHD. The HR for death (1.39) was virtually the same when further adjusting for PVD and CeVD (table 2). Both systolic and diastolic blood pressure as well as serum albumin, phosphorus and 
Table 2. Effect of vascular comorbidities on survival of type 2 diabetic patients starting RRT in 2000-2008 in Finland

\begin{tabular}{lllll}
\hline Vascular comorbidity & $\begin{array}{l}\text { Unadjusted } \\
\text { RR }(95 \% \mathrm{CI})\end{array}$ & $\begin{array}{l}\text { Adjusted }^{\mathrm{a}} \\
\text { RR }(95 \% \mathrm{CI})\end{array}$ & $\begin{array}{l}\text { Adjusted }^{\mathrm{b}} \\
\text { RR (95\% CI) }\end{array}$ & $\begin{array}{l}\text { Adjusted }^{\mathrm{c}} \\
\text { RR (95\% CI) }\end{array}$ \\
\hline CHD & $1.79(1.49-2.15)$ & $1.48(1.22-1.79)$ & $1.39(1.14-1.71)$ & $1.38(1.07-1.78)$ \\
PVD & $1.93(1.59-2.34)$ & $1.88(1.55-2.28)$ & $1.75(1.43-2.14)$ & $1.65(1.29-2.11)$ \\
CeVD & $1.48(1.17-1.88)$ & $1.39(1.10-1.76)$ & $1.09(0.85-1.40)$ & $1.02(0.76-1.35)$ \\
\hline
\end{tabular}

$\mathrm{RR}=$ Risk ratio.

${ }^{a}$ Adjusted for age and gender. ${ }^{b}$ Adjusted for age, gender and the two other vascular comorbidities. ${ }^{c}$ Adjusted for age, gender, the two other vascular comorbidities, systolic blood pressure, diastolic blood pressure, serum albumin, phosphorus, and C-reactive protein.

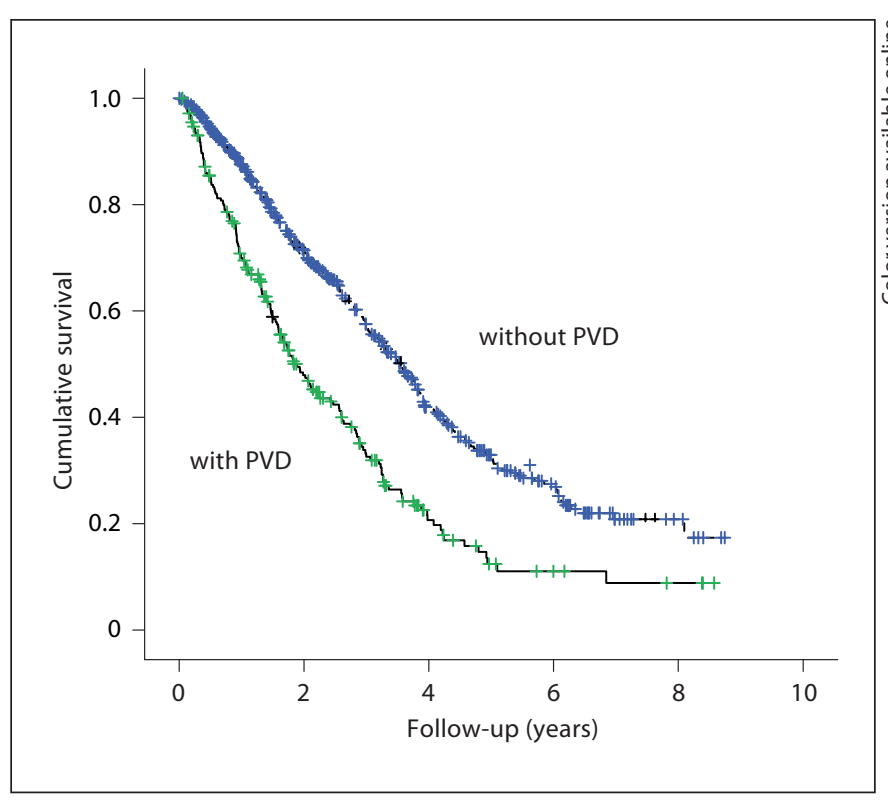

Fig. 1. Survival of diabetes type 2 patients at start of RRT with and without PVD in 2000-2008 in Finland.

CRP at start of RRT were associated with survival, and were considered as potential confounders. Further adjustment for the aforementioned variables did not, however, change CHD patients' HR for death (table 2). HbAlc, serum total or HDL cholesterol or triglycerides, hemoglobin, pulse pressure, previous or current smoking or BMI showed no effect on survival and were not adjusted for.

The median survival was 1.9 years of patients with PVD and 3.6 years of patients without PVD (log rank $\mathrm{p}<$ 0.001 , fig. 1). The median survival was 1.9 years for symptomatic PVD patients, 1.8 years for patients with surgery because of PVD and 1.8 years for patients with limb am- putation. Hardly any patient with PVD received a kidney transplant. The patients with PVD had significantly more CeVD (26 vs. $12 \%, \mathrm{p}<0.001$ ), CHD (57 vs. $37 \%, \mathrm{p}<0.001$ ) and heart failure ( 31 vs. $16 \%, \mathrm{p}<0.001$ ), and were more often previous smokers ( 45 vs. $35 \%, \mathrm{p}=0.01$ ) compared to patients without PVD. Data on vascular access for hemodialysis were available at end of first calendar year of RRT. Of all 877 patients, 798 were alive and 634 on hemodialysis at the end of first calendar year, and data on vascular access were available for 541 (85\%) of these patients. Of the hemodialysis patients, $84 \%$ had a fistula, $2 \%$ a graft and $14 \%$ a central venous catheter, which was more common among PVD patients than others (20 vs. $11 \%, \mathrm{p}=$ 0.021 ), while other vascular comorbidities were not associated with type of vascular access. Because information on vascular access was available only for hemodialysis patients, it was not used for adjustment in survival analysis. When adjusted for age and gender, patients with PVD had an HR for death of 1.88, and the HR remained significantly greater than one with further adjustment for the other vascular comorbidities, blood pressure and laboratory variables (table 2). Of patients with symptomatic PVD, $62 \%$ also had CHD and 27\% had CeVD. These figures were rather similar but somewhat lower in patients with surgery because of PVD (59\% had CHD and 20\% had CeVD) and in patients with limb amputation (41\% had CHD and 24\% had CeVD).

The median survival of patients with CeVD was 2.4 years and without CeVD 3.3 years $(\log$ rank $\mathrm{p}=0.001)$. Patients with CeVD had an age- and gender-adjusted HR for death of 1.39, but with more extensive adjustment the HR did not differ significantly from one (table 2).

Only 43 patients $(6 \%)$ had all three vascular diseases, whereas 311 patients (40\%) did not have any of them at start of RRT (table 3). Of all patients, 275 (35\%) had only one, and 146 (19\%) had two vascular diseases at start of 
Table 3. Combined effect of vascular comorbidities on the survival of type 2 diabetic patients starting RRT in 2000-2008 in Finland

\begin{tabular}{lrrll}
\hline $\begin{array}{l}\text { Combination of } \\
\text { vascular comorbidities }\end{array}$ & $\begin{array}{l}\text { Pa- } \\
\text { tients }\end{array}$ & Deaths & $\begin{array}{l}\text { Median } \\
\text { survival } \\
\text { time, years }\end{array}$ & RR (95\% CI) \\
\hline No comorbidities & 311 & 137 & 4.3 & 1 \\
CHD & 160 & 104 & 2.8 & $1.63(1.25-2.13)$ \\
PVD & 82 & 52 & 2.6 & $2.05(1.49-2.83)$ \\
CeVD & 33 & 20 & 2.2 & $1.85(1.16-2.97)$ \\
CHD + PVD & 93 & 69 & 1.7 & $2.84(2.11-3.83)$ \\
CHD + CeVD & 34 & 21 & 2.7 & $1.65(1.04-2.63)$ \\
PVD + CeVD & 19 & 12 & 2.6 & $2.86(1.57-5.18)$ \\
CHD + PVD + CeVD & 43 & 29 & 1.6 & $2.17(1.44-3.26)$ \\
\hline
\end{tabular}

$R R$ adjusted for age and gender.

RRT. Acute myocardial infarction and its complications, acute ischemic heart disease, sudden cardiac arrest or cardiac lethal arrhythmia was the cause of death in $19 \%$ of patients without any vascular diseases, in $23 \%$ of patients with only one vascular disease and in $31 \%$ of those with two or more vascular diseases. The median survival of the patients with all three vascular diseases was 1.6 years $(95 \%$ CI $0.7-2.5)$ and without any of them 4.3 years (95\% CI 3.8-4.8, log rank $\mathrm{p}<0.001$, fig. 2). Table 3 displays the effect of vascular diseases alone and in combination on survival. Notably, the HR for death was highest in patients with the combination of PVD and either CHD (HR for death 2.84, 95\% CI 2.11-3.83) or CeVD (HR 2.86, 95\% CI 1.57-5.18) when adjusted for age and gender.

\section{Discussion}

PVD predicts worse survival on RRT. CHD or CeVD at start of RRT are also associated with increased mortality, but to a lesser extent. The fact that vascular comorbidities increase mortality is not unexpected, but it has not been known to what extent they impair survival of type 2 diabetes patients on RRT. In this population, vascular comorbidities are important as they are very common, the most frequent being CHD followed by PVD. At the start of RRT, $60 \%$ of the patients had at least one vascular comorbidity, and presence of these comorbidities was connected with a 2- to 3 -fold risk of death. Compared to the study by Helve et al. [9] on type 1 diabetes, our study population of type 2 diabetes had more vascular comorbidities at the start of RRT, for instance CHD was

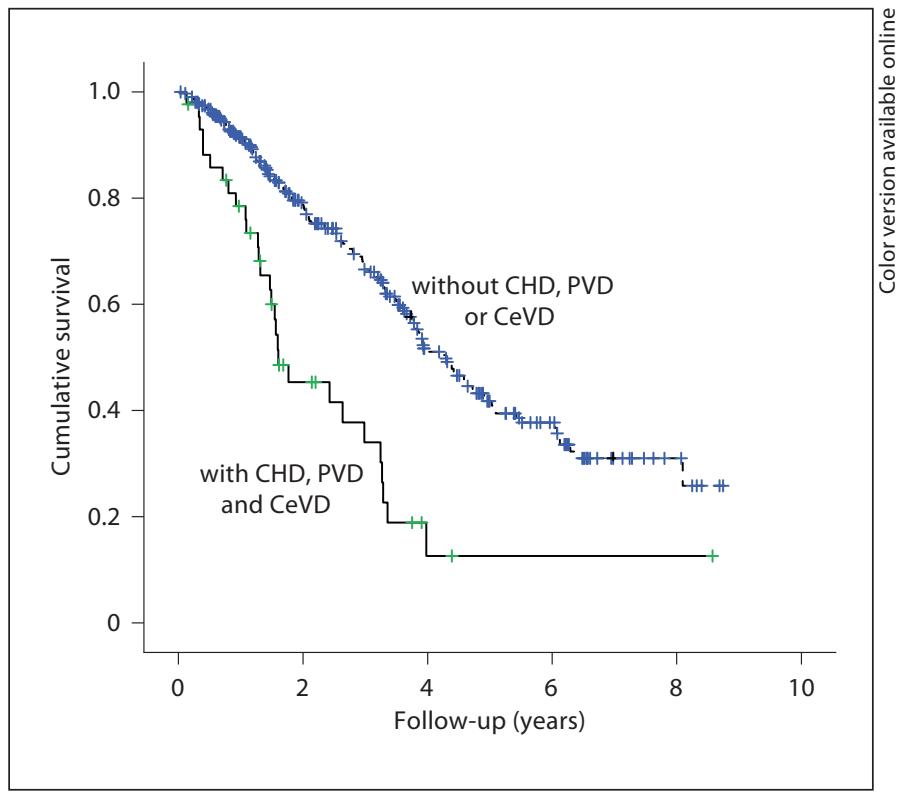

Fig. 2. Survival of diabetes type 2 patients at start of RRT without vascular comorbidities and with all three vascular comorbidities (CHD, PVD and CeVD) in 2000-2008 in Finland.

almost twice as common in our study. Heart failure was also more common in our study (18 vs. $7 \%$ ).

Vascular calcification begins early in the natural history of diabetes, and various consequences of hyperglycemia, such as increased glycation and oxidative stress and glycosylation end products, are associated with calcification in both non-uremic and uremic patients [1113]. Uremia in itself and longer duration of dialysis treatment also increase vascular calcification. Vascular calcification has been shown to be an actively regulated process where both inflammation and osteogenic regulation appear to be involved in its development $[14,15]$.

Aortic stiffness and vascular calcification clearly predict mortality in dialysis patients. Preventive means such as tobacco cessation, hypertension treatment and good blood glucose control should preferably be introduced early, already at the time when blood glucose impairment is detected. Recently, results from a large screening study have shown that more than two thirds of patients with chronic kidney disease have at least one modifiable major risk factor for cardiovascular diseases that could be improved [16]. In our study, past smoking was more common among patients with vascular comorbidities, but smoking itself did not affect survival. Thus, attention should be paid to supporting these patients to quit smoking long before they enter RRT [17]. A risk factor that had 
an effect on survival among the patients in our study was serum phosphorus, the level of which can be decreased with medication. A low serum albumin also had a strong impact on risk of death, most probably as a marker of poor nutritional status.

Results of coronary bypass surgery have not been as favorable in diabetes patients as in others [18]. Outcomes of coronary angioplasty have improved greatly, and this is true also for patients with diabetes thanks to the introduction of drug-eluting stents. Still, diabetes patients more often need repeat interventions [19]. In a recent study by Sunagawa et al. [20], the long-term outcomes of coronary artery bypass grafting were superior to angioplasty with drug-eluting stents in patients with chronic renal failure on hemodialysis. Revascularization outcomes for PVD have been poor in ESRD [21], and amputations are especially frequent among hemodialysis patients with diabetes [22]. In our study, patients with a history of amputation or surgery because of PVD had similar survival probability compared to patients with PVD.

An important strength of our study is the nationwide virtually $100 \%$ coverage of all patients entering RRT due to type 2 diabetes in Finland during the study period. This reduces selection bias. Data on the main outcome death are also complete. A very large proportion of the patients had information on all comorbidities studied. Confounding could be effectively controlled thanks to abundant information on possible confounding factors. In contrast to most of the earlier studies which have studied both type 1 and type 2 diabetes, we were able to exclude patients with type 1 diabetes.
In the past few years, the incidence of ESRD due to type 2 diabetes has stopped increasing [10]. One reason for this may be the improved management of diabetes. On the other hand, it should be noted that high mortality before developing severe kidney injury can also explain low incidence of ESRD. Cardiovascular risk factors and preventive measures during the early phase of type 2 diabetes are well known. Further study is needed to establish the role of various risk factors among type 2 diabetes patients who have developed ESRD. Our study showed that vascular comorbidities and especially PVD impair survival. This stresses the importance of preventing atherosclerosis and slowing down development of vascular calcifications. Such prevention must be introduced long before RRT starts. When the type 2 diabetes patient has developed ESRD, adequate dialysis and treatment of hyperphosphatemia are probably the most important means to improve the very poor outcome [23, 24]. Cardiac interventions may be associated with improved long-term outcomes in chronic kidney disease patients [25], but it is not known how various cardiac or vascular interventions affect long-term mortality in patients with ESRD. In conclusion, peripheral arterial disease is the strongest predictor of death when patients have survived until RRT for type 2 diabetes-associated nephropathy.

\section{Disclosure Statement}

None.

\section{References}

1 Blacher J, Guerin AP, Pannier B, Marchais SJ, London GM: Arterial calcifications, arterial stiffness, and cardiovascular risk in endstage renal disease. Hypertension 2001;38: 938-942.

$\checkmark 2$ Foley RN, Culleton BF, Parfrey PS, et al: Cardiac disease in diabetic end-stage renal disease. Diabetologia 1997;40:1307-1312.

-3 London GM, Guérin AP, Marchais SJ, Métivier F, Pannier B, Adda H: Arterial media calcification in end-stage renal disease: impact on all-cause and cardiovascular mortality. Nephrol Dial Transplant 2003;18:17311740.

-4 Shoji T, Emoto M, Shinohara K, et al: Diabetes mellitus, aortic stiffness, and cardiovascular mortality in end-stage renal disease. J Am Soc Nephrol 2001;12:2117-2124.
-5 Adler AI, Stevens RJ, Manley SE, Bilous RW, Cull CA, Holman RR, UKPDS GROUP: Development and progression of nephropathy in type 2 diabetes: the United Kingdom Prospective Diabetes Study (UKPDS 64). Kidney Int 2003;63:225-232.

-6 Keith DS, Nichols GA, Gullion CM, Brown JB, Smith DH: Longitudinal follow-up and outcomes among a population with chronic kidney disease in a large managed care organization. Arch Intern Med 2004;164:659663.

7 Villar E, Polkinghorne KR, Chang SH, Chadban SJ, McDonald SP: Effect of type 2 diabetes on mortality risk associated with end-stage kidney disease. Diabetologia 2009; 52:2536-2541.
${ }_{8}$ Lok CE, Oliver MJ, Rothwell DM, Hux JE: The growing volume of diabetes-related dialysis: a population based study. Nephrol Dial Transplant 2004;19:3098-3103.

\9 Helve J, Haapio M, Groop PH, GrönhagenRiska C, Finne P: Comorbidities and survival of patients with type 1 diabetes on renal replacement therapy. Diabetologia 2011;54: 1663-1669.

10 Finnish Registry for Kidney Diseases. Annual Report 2008. Helsinki, Finnish Registry for Kidney Diseases, 2009. http://www.musili.fi/smtr/english.

-11 Ishimura E, Okuno S, Kitatani K, et al: Different risk factors for peripheral vascular calcification between diabetic and non-diabetic haemodialysis patients - importance of glycaemic control. Diabetologia 2002;45:14461448 
12 Kilhovd BK, Juutilainen A, Lehto S, et al: Increased serum levels of advanced glycation endproducts predict total, cardiovascular and coronary mortality in women with type 2 diabetes: a population-based 18 year follow-up study. Diabetologia 2007;50:14091417.

13 Ishimura E, Okuno S, Taniwaki H, et al: Different risk factors for vascular calcification in end-stage renal disease between diabetics and nondiabetics: the respective importance of glycemic and phosphate control. Kidney Blood Press Res 2008;31:10-15.

- 14 Jono S, Shioi A, Ikari Y, Nishizawa Y: Vascular calcification in chronic kidney disease. J Bone Miner Metab 2006;24:176-181.

-15 Shao JS, Cheng SL, Sadhu J, Towler DA: Inflammation and the osteogenic regulation of vascular calcification: a review and perspective. Hypertension 2010;55:579-592.

16 McCullough PA, Steigerwalt S, Tolia K, et al, KEEP Investigators: Cardiovascular disease in chronic kidney disease: data from the Kidney Early Evaluation Program (KEEP). Curr Diab Rep 2011;11:47-55.
17 Fagard RH: Smoking amplifies cardiovascular risk in patients with hypertension and diabetes. Diabetes Care 2009;32(suppl 2): S429-S431.

18 Cook S, Windecker S: Revascularisation of coronary artery disease in patients with diabetes mellitus. Swiss Med Wkly 2009;139: 654-664.

19 Moussa I, Leon MB, Baim DS, et al: Impact of sirolimus-eluting stents on outcome in diabetic patients: a SIRIUS (SIRolImUS-coated Bx Velocity balloon-expandable stent in the treatment of patients with de novo coronary artery lesions) substudy. Circulation 2004;109:2273-2278.

20 Sunagawa G, Komiya T, Tamura N, Sakaguchi G, Kobayashi T, Murashita T: Coronary artery bypass surgery is superior to percutaneous coronary intervention with drug-eluting stents for patients with chronic renal failure on hemodialysis. Ann Thorac Surg 2010; 89:1896-1900
21 Reddan DN, Marcus RJ, Owen WF Jr, Szczech LA, Landwehr DM: Long-term outcomes of revascularization for peripheral vascular disease in end-stage renal disease patients. Am J Kidney Dis 2001;38:57-63.

22 Combe C, Albert JM, Bragg-Gresham JL, et al: The burden of amputation among hemodialysis patients in the Dialysis Outcomes and Practice Patterns Study (DOPPS). Am J Kidney Dis 2009;54:680-692.

23 The FHN Trial Group: In-center hemodialysis six times per week versus three times per week. N Engl J Med 2010;363:2287-2300.

24 Wald R, Sarnak MJ, Tighiouart H, et al: Disordered mineral metabolism in hemodialysis patients: an analysis of cumulative effects in the hemodialysis (HEMO) study. Am J Kidney Dis 2008;52:531-540.

25 Appleby CE, Ivanov J, Lavi S, et al: The adverse long-term impact of renal impairment in patients undergoing percutaneous coronary intervention in the drug-eluting stent era. Circ Cardiovasc Interv 2009;2:309-316. 